\title{
INSTRUMENTATION AND DATA ACQUISITION SYSTEMS
}

\author{
Joseph F. Guarino \\ NASA Langley Research Center
}

\author{
INTRODUCTION
}

The National Transonic Facility (NTF), with its unique operating characteristics, imposes new and demanding requirements on instrumentation and measurement systems. Cryogenic operation - a new dimension in wind-tunnel testing - high dynamic pressure, and the need for fast data rates are factors which individually or collectively affect virtually all instrument designs and, in some cases, dictate new approaches. Furthermore, the uniqueness of this facility also dictates the need for an extensive tunnel calibration and process monitoring system. Over 2000 individual measurements have been identified to date; these measurements include the traditional tunnel survey and flow-quality measurements and the monitoring of critical facility systems and components.

To meet these requirements, a comprehensive and integrated measurement system has been identified and a design and development effort has been initiated to meet the criteria imposed by the NTF operating environment. Specific measurement areas receiving concentrated attention include: data acquisition, force measurement, pressure instrumentation, flow-visualization techniques, model attitude and model deformation measurement, and temperature measurement. A description of the ongoing work in each of these areas is presented with particular emphasis on the approaches being considered and the status of the current work.

\section{NTF INSTRUMENT COMPLEX}

\section{Introduction}

The NTF instrument complex (fig. 1) will be centered around four 32-bit, 1-microsecond-cycle-time central processing units connected in a multipointdistributed network configuration. The principal activities to be supported by these computers are (1) data base management and processing, (2) research measurement data acquisition and display, (3) tunnel and model control, and (4) process monitoring and communication control. The distributed network approach has been chosen to modularize the functional software into definable and implementable parts by the various groups involved in the design and to permit use of similar hardware configurations to improve reliability and maintainability. This design can allow essential processes to continue when primary hardware failures occur by central processing unit (CPU) substitution and elimination of 
desirable but nonessential activities. Research measurement data acquisition and tunnel and model control are identified as essential functions.

Data Base Management and Central Processing Unit (CPU)

This $256 \mathrm{~K}$ byte main memory computer will control all standard peripheral equipment. It will maintain master files of all processors, library routines, user application programs, archival data for comparison, and logical files of information for the other three computers.

Part of its memory will be shareable and organized so that it can function similar to common memory in a conventional computer system. The $32 \mathrm{~K}$-byteshared memory will be more powerful in that read and write control can be selectively exercised from the various other computers. For example, part of this shared memory will be designated as writeable from the research measurement data-acquisition computer. Acquired research measurements will be stored in this shared memory for use by the other computers; however, this part of memory will be read only from the other computers. In this way, the data management CPU can use the data for plotting, listing, etc., while the tunnel control CPU uses the same measurement in control calculations without the possibility of destroying the original value.

Job-entry control will be by means of a standard alphanumeric cathode ray tube (CRT). Two card readers operating at 1000 cards per minute will allow input of logical files to be used by this CPU or passed to the various other CPU's.

Display devices will include two 1000 lines-per-minute line printers. An interactive 10- by 15-inch plotter will provide report quality graphs. A 19inch interactive graphic CRT will provide online presentation of test progress and will include hardcopy output and floppy-disk storage for retention and comparison of test results.

Recording devices will include three magnetic tape units. These units will be nine-track $800 / 1600$ bits per inch with recording speeds of $60 \mathrm{~K}$ to $120 \mathrm{~K}$ bytes per second. Three types of disk units will be used to cover the various mass storage requirements. Floppy disks will be used to conveniently store user source program files and provide compatibility with CRT plot data files. A medium-capacity high-transfer-rate cartridge disk will be compatible with the disk files used on the other three processors. Storage of 25 megabytes with transfer rates of $200 \mathrm{~K}$ bytes per second will be typical for these units. The third disk will be the large capacity archival type with over 100 megabytes capacity.

In addition to the shared memory communication with the other processors, a standard input/output ( $\mathrm{I} / 0$ ) channel interface or communication channel interface will exist to permit multipoint intercommunication of the systems. This will allow various levels of communications in the software structure to improve system reliability. 
The data management CPU will also include an interface to the new highspeed pressure measurement system to provide recording and processing of these measurements.

\section{Data Acquisition and Display Central Processing Unit (CPU)}

The data acquisition and display CPU, as well as the tunnel and mode1 control and process monitoring and communications CPU's, will each include a 32bit, 1-microsecond-cycle-time, 256K-bytes-memory CPU. Each of these three systems will have an alphanumeric console CRT for system control and a mediumcapacity disk unit for program and local data storage. Accumulated data will be transferred from the local disk to the data management system where it will be either recorded directly if a magnetic tape unit is available or temporarily stored on the archival disk. Since each local disk unit will be of the cartridge type, the data can be physically transferred to the data management C.PU and recorded directly after the test, if necessary.

This system will be principally concerned with the management of the 384 research measurements from the tunnel and three static test setup areas. Up to 256 channels will be available to the tunnel test, the remaining 128 channels being shared by the three setup areas. The Data Acquisition Unit (DAU) will acquire data at rates up to 50000 samples per second. Each low-level differential channel will include programable gain ranges from 8 to $64 \mathrm{mV}$ full scale. Antialiasing filters with several different cutoffs from $5 \mathrm{~Hz}$ to $1000 \mathrm{~Hz}$ will be supplied. Automatic calibration for gain and offset will be under software control. Overall inaccuracy is expected to be no more than 0.1 percent.

An interface to a 14-channel FM tape recorder will permit post-run digitization and playback. Function inputs/outputs will be available to provide controls to powered or movable surface models, drive numeric digital indicators with coefficients, run numbers, Mach numbers, etc., and to accept inputs from user-oriented data acquisition control panels.

Tunnel and Model Control Central Processing Unit (CPU)

The CPU, console CRT, and disk for this system will be as described in the section "Data Acquisition and Display Central Processing Unit." The DAU will be similar; however, it will be limited to a tota1 of 64 input channels. The function inputs/outputs will be interfaced with a group of 10 subprocess controllers which will perform the actual closed-loop control. These subprocess controllers are envisioned as either microprocessors or hybrid dedicated controllers and will be used for control of (1) inlet guide vane, (2) model roll and pitch, (3) spoiler flaps, (4) test-section walls, (5) reentry flaps, (6) pressure, (7) temperature, (8) cooling water flow, (9) liquid nitrogen, and (10) drive speed. Additional inputs will be monitored to insure that hardware interlocks and permissives are in order and that safe operation can be achieved.

\section{Process Monitoring and Communication Central Processing Unit (CPU)}

The CPU, console CRT, and disk for this system will be as described previously. The DAU will be much slower - 25 to 100 channels per second. Some inputs will be single-ended $\pm 10 \mathrm{~V}$ full scale whereas others are of the low-level 
differential type. The process variables monitored will include such measurements as tunnel pressure and stress, drive-system temperatures and vibration, power consumption, etc. Function inputs/outputs will drive alarms such as bells and visual displays to indicate out-of-limit conditions.

This system will include'a communication handler to provide access to sources such as the NASA Langley Instrument Research Division for program development, test, and debugging, and the central computer complex at Langley Analysis and Computation Division for transmittal of test data for complete data reduction. Several other synchronous and asynchronous lines will be available at rates from 110 to 9600 baud to connect to any national computing facility using major line protocols supported by IBM, CDC, and Univac. This access is believed to be beneficial to groups scheduling tests which require customized processing by permitting machine access for program development and debugging prior to the actual scheduled test. It can also be used to return unclassified data to outside sources concurrent with the ongoing test.

\section{FORCE INSTRUMENTATION}

The National Transonic Facility tunnel imposes rather severe requirements on the measurement of aerodynamic forces and moments. Not only does the cryogenic environment present an unusual surrounding for the force balances but also, because of the tunnel's high density capability, the magnitude of the load to be measured is about five times that of a conventional tunnel. Although extending the state of the art, initial studies indicate that a family of six-component high-capacity force balances can be built to satisfy NTF requirements. To cope with the cryogenic environment, thermal protection in the form of a "heat jacket" or resistance heating was considered; however, early investigations indicated that strain-gage balances behave predictably in the cryogenic environment without thermal control.

\section{Balance Loads}

The maximum balance loads to be experienced from a typical transport model have been estimated as follows:

\begin{tabular}{|l|r|r|}
\hline \multicolumn{1}{|c|}{ Component } & \multicolumn{2}{|c|}{ Load } \\
\hline Normal force N(1b) & 86736 & $(19500)$ \\
Axial force N(1b) & 6939 & $(1560)$ \\
Pitch moment Nm(in-1b) & 2937 & $(26000)$ \\
Roll moment Nm(in-1b) & 1762 & $(15600)$ \\
Yaw moment Nm(in-1b) & 1762 & $(15600)$ \\
Side force N(1b) & 17347 & $(3900)$ \\
\hline
\end{tabular}

These loads represent the upper extreme of the NTF operating envelope and will seldom be reached; however, a preliminary balance design test was performed that indicates an $8.89-\mathrm{cm}$ diameter $(3.5-\mathrm{inch})$ configuration shown in figure 2 
could satisfy these requirements with a maximum stress level of less than $689 \mathrm{MN} / \mathrm{m}^{2}$ (100 $000 \mathrm{psi}$ ). A high-strength alloy steel will be utilized to provide a safety factor of approximately 2.5 based on the yield strength. Since it is unlikely that all listed loads would be experienced at one time, realistically the balance safety factor is greater than 2.5.

A curve illustrating current Langley Research Center (LaRC) high-capacity balance capability is shown in figure 3. A plot of balance normal-force capacity against balance diameter is given; this plot is based on the assumption that all six components are loaded simultaneously. Obviously, if some of the components are reduced, the normal-force capacity can be increased even further. Two immediate consequences of going to extremely high capacity balances are increased balance deflections for a given size and more critical or demanding calibration procedures. With increasing deflections, second-order interactions become more pronounced and make it imperative that cross-1oad combinations be applied in the calibration procedure. Evaluation of all second-order terms has long been a Langley Research Center policy; therefore, this procedure presents no new requirement.

\section{Temperature Considerations}

When the Langley 0.3 -meter transonic cryogenic tunnel became operational, an electrical resistance heated strain-gage balance was designed and used to measure the aerodynamic loads. Since water-cooled balances have long been used successfully in high-temperature facilities, it was logical to use a heated balance in a cold facility. Five heating stations with feedback sensors were located along the length of the balance to eliminate gradients. During the actual tunnel runs, it was found to be extremely difficult to maintain or control temperature throughout the balance because the heat-transfer rate changed drastically with angle of attack. Tunnel runs were made at several balance control temperatures; some of the results are shown in figure 4. These data indicate that less balance differential temperature is experienced when no thermal control is used and that, in general, the runs all fell close, that is, within the +0.5-percent full-scale uncertainty quotation of the balance. These loads were measured, however, at the low end of the balance design range; therefore, the percent uncertainty would be greater when referenced to the applied load.

The data obtained in the $0.3-\mathrm{m}$ transonic cryogenic tunnel tests are only part of a more comprehensive balance evaluation and development effort. Severa1 projects are underway to evaluate candidate cements and strain-gage bonding procedures, solders, strain gages, and balance materials for cryogenic operation. Data obtained from standard test beams loaded at room temperature as a baseline, and at reduced temperatures are used to evaluate these factors. In one rather severe test, a beam was loaded while at room temperature and was submerged in liquid nitrogen. The results indicated a decreased output (sensitivity) at the lower temperature as expected; but, more importantly, the data were linear with load and displayed no hysteresis or zero shift. In addition, a conventional balance has been evaluated at various temperatures from $297 \mathrm{~K}$ to $77 \mathrm{~K}\left(+75^{\circ} \mathrm{F}\right.$ to $-320^{\circ} \mathrm{F}$ ). The results (fig. 5) again show a decrease in component sensitivity at reduced temperatures and a slight change in the two interactions from their room-temperature value. The variation in interactions is attributed to 
temperature differentials in the balance resulting from a less than ideal test setup. Here again, the balance performed very well and nothing from the test seems to preclude the use of balances at cryogenic temperatures. To compensate for the reduced sensitivity, work is being performed to better match the straingage characteristics with the changing balance material modulus.

In addition, balance material is also being investigated. The usual balance materials, 18-percent nickel grade 300 maraging steel and $17-4 \mathrm{PH}$ stainless steel, each have relatively low fracture toughness and impact strengths in the cryogenic region. Some of the candidate materials and their characteristics are shown in figure 6. Maraging steel grade 200 and 250 appear to have high yield strengths and also good impact qualities. Several of the candidate materials will be evaluated and instrumented with selected gages for testing over the NTF operating temperature range.

\section{MODEL PRESSURE MEASUREMENTS}

Because of the high operating cost of the NTF wind tunne1, pressure measurements will be taken in either a fast "pitch-pause" sequence or a continuouspitch mode. To accommodate these modes of operation, a pressure measurement system with a high data rate is required. This requirement for a high data rate rules out the use of conventional electromechanically scanned pressure sampling techniques and makes it necessary to use individual pressure sensors placed in the model to eliminate long tube lengths and their corresponding slow pressure response. The use of commercially available individual pressure sensors is often impractical because (1) pressure sensors that have the accuracy needed are too large, (2) pressure sensors that are small enough to be placed in the model (such as the semiconductor pressure sensors) do not have the required accuracy, and (3) the cost of individual transducers is sometimes prohibitive when hundreds of pressure measurements are needed. These problems have prompted the Langley Research. Center to develop for the NTF (and its other wind-tunnel facilities) a pressure sensor module that largely overcomes the above-mentioned drawbacks of size, accuracy, and cost. This module utilizes miniature silicon diaphragm pressure sensors, miniature electronic multiplexers, a miniature multiport, and a pneumatically operated pressure selector switch to achieve small size, high data rate, and high accuracy through a full in situ calibration capability.

A photograph of the first generation of these pressure sensor modules is shown in figure 7. This module consists of a substrate containing 16 solidstate pressure-sensor chips and signa1, multiplexing electronics (shown in figure 8) mounted to a four-position pressure selector switch. The use of silicon diaphragm pressure sensor chips as the pressure-sensing elements allows the sensor substrate to be made very sma11 $(4.3 \mathrm{~cm}$ by $2.48 \mathrm{~cm}$ by $0.1 \mathrm{~cm})$ and at the same time give high sensitivity. By using miniature electronic multiplexers to sample the sensor's analog output, the sensor's output can be electronically scanned at a high data rate (in excess of 50000 measurements per second) and large numbers of wires are eliminated. The pressure selector switch occupies most of the volume of the sensor module but is absolutely necessary in order that a full in situ calibration of each sensor be made. Thus, corrections 
for zero and sensitivity shifts usually associated with these semiconductor sensors can be made and an acceptable accuracy be obtained. This firstgeneration pressure sensor module, in addition to the calibration position, can be switched to three "use" positions where 16 sets of pressure ports can be measured. This scheme allows 48 pressure ports to be measured with 16 sensors by pneumatically multiplexing the input pressure ports to the sensor substrate board. However, when all 48 channels are used, a data rate of only approximately 800 samples per second (SPS) is possible, because it takes about 20 milliseconds to pneumatically multiplex the pressure selector switch. When it is used as a 16-channel pressure sensor module, only two positions of the pressure selector switch, the calibrate and one "use" position are used. This operating mode allows the sensors to be calibrated in less than 1 second and then switch to one of the "use" positions where the 16 sensors can be read out at a rate of greater than 50000 SPS. The use of the pressure sensor module for 16 measurements rather than 48 allows a much higher data rate, but causes. a threefold increase in sensor volume per channel.

The design for this first-generation general-purpose pressure sensor module was adopted after considering tradeoffs between size, data rate, accuracy, part count, repairability, cost, and reliability with the constraint that only existing and readily available technology be employed. A second generation of these pressure sensor modules is planned for use in the NTF. These modules will have only a two-position selector switch - one position for calibration and one for measurements of unknown pressures. The pressure selector switch* for a 16-channel module that has been developed is rough1y one-third the size $(1.2 \mathrm{~cm}$ by $2.5 \mathrm{~cm}$ by $1.5 \mathrm{~cm})$ of the first pressure switch*. The sensor substrate for this module will be of a monolithic design with the 16 sensors etched into a silicon substrate $1.2 \mathrm{~cm}$ by $2.5 \mathrm{~cm}$ by $0.05 \mathrm{~cm}$ in dimension. Modules of this design can be ganged together to provide as many channels as necessary.

The large number of sensors involved, the high data rate required, and the need for controlling and addressing of the pressure sensor modules have also prompted LaRC to develop a special-purpose, computer-oriented data acquisition and control system for use with these modules. A block diagram of this pressure measurement system (PMS) is shown in figures 9 and 10 . It is essentially a stand-alone computer-based data acquisition and processing system that is treated as a peripheral for pressure measurements by the central computer. The PMS is controlled by the researcher (through the central computer) by an instruction set that allows various modes of operation (each with several options) to be selected. The PMS is also modular and thereby allows the system to be expandable from a minimum size of 256 ports with $25 \mathrm{~K}$ data storage capacity to a maximum size of 3584 ports through the use of 14 data acquisition and control units (DACU), each with 25K of storage. The maximum data rate of each DACU is 50000 SPS. Higher system data rates can be achieved by parallel operation of the DACU's. The DACU's also have a preprocessing capability that allows data averaging and statistical analysis to be performed before the data is transferred to the central computer.

*Pressure selector switches were developed for Langley Research Center by the Scanivalve Corporation, San Diego, CA. 
Various parameters for the PMS are shown in figure 11 for 300 and 1000 pressure-port operation. For the 300 pressure-port operation, two DACU's, each utilizing 150 ports would be used. This configuration allows 166 measurements per port to be taken in 0.5 second at a data rate of 100000 SPS (twice the basic data rate of 50000 of the DACU since two DACU's are being used) for a total of 50000 measurements stored in the two DACU's. Time between measurements on the same port is 3 milliseconds. For the pitch-pause mode of operation with a 2.5-second period for each pitch-pause sequence, the data would be averaged and the standard deviation computed during the pitch phase; these 333 data points will be transmitted to the central computer for display and analysis storage. For a 1000 pressure-port operation, four DACU's, each utilizing 250 ports, would be used. This configuration allows 100 measurements per port to be taken in 0.5 second at a system data rate of 200000 SPS. for a total of 100000 data points. The lapse time between measurements on the same port is 5 milliseconds. As before, these data would be averaged and the standard deviation computed during the pitch cycle, and the results transmitted back to the central computer. In both the 300- and 1000-port operation, the electronic scanning pressure (ECP) modules would be calibrated before and after each polar.

For a continuous pitch operation, the sensor modules would also be calibrated before and after each polar. Calibration coefficient data and the raw pressure measurement data for the continuous-pitch mode of operation would be transferred from the DACU's after each polar to the central computer for processing, display, analysis, and storage, since the PMS has a limited processing capability. This data transfer would take less than 1 second. For the 300and 1000-port operation, a total of 166 and 100 pressure measurements per port, respectively, would be taken for each polar. Selected pressure measurements can be displayed during the test.

The Langley Research Center is developing a specia1-purpose pressuremeasurement system that will allow measurement rates several orders of magnitude greater than those currently obtainable with electromechanical pressure sampling type and with no loss of measurement accuracy. This system is characterized by in situ calibration, small size, high data rate, stand-alone operation, modular design, and a user-oriented software instruction set. This system will allow either pitch-pause or continuous-pitch operation.

\section{FLOW VISUALIZATION}

The current NTF design does not include any flow-visualization systems for initial operation. However, to insure the ability to incorporate such systems in the future, work is underway on the conceptual design of a schlieren system and a laser velocimeter system compatible with tunnel design constraints.

The principal constraint on the schlieren system design is the requirement that it be completely contained within the tunnel plenum. Because of the cryogenic environment, the schlieren system components will be housed in environmentally controlled cylinders mounted on each side of the test section. The design will permit the cylinders to be traversed for alinement and desired 
viewing positions. Conceptually, the present design (fig. 12) uses $61 \mathrm{~cm}-$ diameter (24-inch) f/5 parabolic mirrors. The cylinders will be insulated and the internal pressure equalized with the plenum static pressure. Positioning of the elements of the system will be accomplished by remote control. Quartz windows with diameters of $61 \mathrm{~cm}$ (24 inch) will be used in the cylinders. These windows will be subjected to severe thermal shock and must function with a large temperature gradient across them without degrading the quality of the schlieren image. An experimental program, utilizing a specially designed test apparatus, is currently underway to investigate these effects. Future activities will include study of component alinement requirements, vibration and mounting effects, and insulation and temperature control requirements. The end product of these studies will be the design criteria required for developing a final schlieren system design for NTF.

Similar design constraints are imposed on an operational laser velocimeter (LV) system for NTF. Mounting of the system components in environmenta1ly controlled cylinders as described for the schlieren system will be required. The LV system installed in the schlieren system cylinder will provide measurements of the $\bar{u}-$ and $\bar{v}$ - velocity components. Obtaining the $\bar{u}$ components presents a major problem. The problem arises because the presently envisioned method for obtaining this measurement requires an additional laser-optics package, containment cylinder, supporting mechanisms, and viewing ports in the overhead or floor area of the test. section. This location is prohibited and therefore an alternate scheme must be developed. Also of primary concern, and an item which will be investigated is the development of a suitable and acceptable "seeding" technique compatiblewith the cryogenic flow medium in NTF.

\section{MODEL ATTITUDE}

The traditionally difficult model-attitude measurement problem is compounded by the cryogenic environment in NTF, the need for fast response for efficient tunnel operation, and the new requirement for measuring both pitch and roll angles simultaneously as dictated by the two-degree-of-freedom sting motion. Two basic approaches are being pursued for this measurement. These include the application of inertial sensors, which is the most common technique employed at LaRC and elsewhere, and the development of an optical angle sensing system.

The inertial sensor approach would require three sensors encased in an environmentally controlled container to be mounted in the model to provide both pitch and roll sensing. Also included would be a precision electrolytic bubble sensor to serve as a "zero" reference. This approach is straightforward and relies on existing and known techniques. However, the principal disadvantages of this approach include the relatively large size, the slow signal-response characteristics (resulting from required heavy signal filtering), and limited accuracy due to susceptibility of the inertial sensor to vibration.

Optical techniques show a great deal of promise for model attitude measurement. Their principal advantages include: fast response, high resolution and accuracy, insensitivity to vibrations, and remote sensing capability 
requiring only minor model space or interference. Two basic approaches are under study. The first employs a differential photocell mounted in the model surface and illuminated by a laser light source located in the plenum. It may be possible to configure the optical sensor to measure angles about both axes; thus, the simultaneous pitch-roll measurement requirement is satisfied. Future work will be directed toward developing techniques for increasing the current range from $\pm 5^{\circ}$ to the required values in NTF $\left(+45^{\circ}\right)$ and to developing temperature-tolerant photosensors or thermal protection techniques. A second, and very promising approach, is basically an interferometric system offering very high accuracy, good range, and very fast response characteristics. On1y a passive reflector is required on the model, most of the optics and electronics being located in the plenum. This approach differs from other interferometric systems in that it is basically insensitive to tunnel-flow perturbations. A private firm is currently working on a proof-of-concept system at Langley's request. A test will be conducted in the near future, the results of which will determine future effort and the required funding to develop a prototype system.

\section{MODEL DEFORMATION MEASUREMENT}

Model deformation measurements at Langley have in the past been restricted to double exposure photography. (See ref. 1.) More recently, stereophotography has been used at the Langley 8-foot transonic pressure tunnel for wingdeformation measurements with an accuracy of about $0.125 \mathrm{~mm}$. Other techniques which have been advocated such as interferometry (both conventional and holographic), geometrical techniques (including Moiré fringe methods), and modulated light-beam methods have had little or no success in actual wind-tunnel tests. Several of the techniques rely heavily on recently developed technology in semiconductor lasers and photodiodes.

Since various groups across the country are actively working on the interferometric and geometrical methods, the effort at Langley has been directed toward the modulated, light-beam technique. In this technique, a light source (such as a laser diode) is amplitude-modulated at microwave frequencies. The phase of the received light reflected from the target mounted on the model is then compared with the phase of the transmitted light to provide displacement. This technique is currently in use in several surveying instruments (ref. 2) but these instruments are neither accurate nor versatile enough for direct application to NTF.

An earlier prototype system at Langley employed a 1ight-emitting diode and an avalanche photodiode (ref. 3). By use of a retroreflector, laboratory displacement measurements were made at a modulation frequency of $1.5 \mathrm{GHz}$ at a range of 1 meter. The present laboratory setup (fig. 13) consists of a commercially packaged laser diode which can be operated as a continuous wave at room temperature. A high-frequency signal from a 50-ohm output impedance signal generator (after impedance matching to the low dynamic impedance of the diode) is used to modulate the output of the laser diode about its dc bias point. A X10 microscope objective is used to quasi-collimate the $8200^{\circ}$ A laser 1 ight. The light is directed to a $90^{\circ}$ prism which folds the beam $180^{\circ}$ and introduces 
a slight spatial offset. The return beam is focused and directed to the active area of the PIN photodiode. Movement of the $90^{\circ}$ prism along the optical axis introduces phase shifts in the return beam which can then be compared with the phase of the outgoing beam. With this system, modulation frequencies of up to $2.4 \mathrm{GHz}$ have been used.

The phase difference has been measured in several ways. The most direct way is to feed a small part of the signal that is used to modulate the laser to one channel of a high-speed dual-trace oscilloscope while feeding the signal from the photodiode to the other channel. By triggering both channels from the reference modulating signal, the phase of the signals can be compared. Phase differences on the order of $3^{\circ}$ can be detected by using this method, which for a 500-MHz modulating frequency corresponds to a resolution of $2.5 \mathrm{~mm}$. The two signals can also be mixed with a dc-coupled mixer. The dc output of the mixer is proportional to the phase difference. Another way to determine the phase is to frequency downshift both the reference and phase-shifted signals (to about $100 \mathrm{kHz}$ ) with a common local oscillator. The phase of the two downshifted signals is then compared with a commercial phasemeter with a resolution of $0.1^{\circ}$. This more accurate means of monitoring phase showed the phase difference between the two beams to drift with time. Both modulator and detector introduce phase shifts which vary with time and temperature (ref. 2). For this reason it is more accurate to measure phase by comparing the modulated reference and signal light beams directly. This can be done by superimposing the two light beams at the detector. Since the instrument is not being operated in an optical frequency interferometric mode, alinement and beam path mismatch are not critical. When the light arriving at the detector from the two beams is in the phase, a maximum output from the detector occurs.

In conclusion, it should be emphasized that while laboratory studies are currently being restricted to the modulated-light method, other techniques may be considered. Comments and suggestions on other remote displacement measurement systems which might be applicable to NTF are invited.

\section{MACH NUMBER MEASUREMENT}

The accurate determination of the operating Mach number in the NTF to within a $\triangle M$ of \pm 0.002 is complicated by very wide pressure range of $35 \times 10^{3}$ to $896 \times 10^{3} \mathrm{~N} / \mathrm{m}^{2}$ (5 to $130 \mathrm{psia}$ ) to be covered and the rapid pressuresensing response necessary to minimize operating costs. In addition, the control of Mach number to within this $\Delta \mathrm{M}$ tolerance requires a pressure measurement uncertainty of no greater than $400 \mathrm{~N} / \mathrm{m}^{2}(0.06 \mathrm{psi})$ when the total pressure is $480 \mathrm{kN} / \mathrm{m}^{2}$ (69.6 psia). However, pressure measurement instrumentation, such as manometers, fused-quartz-type gages, and other types, which can satisfy this measurement requirement suffer from limited frequency response.

Research is underway to evaluate the dynamic characteristics of an electronic mercury manometer and to identify other pressure measurement problems which may affect the performance of the National Transonic Facility. Automation of the NTF tunnel operation can lead to maximizing data production while minimizing tunnel operation cost. To maintain such tunnel parameters as Mach 
number, dynamic pressure, etc., automatic control regulation is necessary. Inherent in the control loop are transducer dynamics as well as the tunnel characteristics. In the case of pressure-related regulation, where required measurement accuracy may be less than $24 \mathrm{~N} / \mathrm{m}^{2}(0.5 \mathrm{psf})$, mercury type manometers are best suited. For the manometer selected, the response to a $2400 \mathrm{~N} / \mathrm{m}^{2}$ (50 psf) pressure change requires about 6 seconds to settle to within the required accuracy limits. In question, then, is how to maintain the accuracy of parameter regulation with a minimum of settling time to required accuracy limits. In this work, a manometer dynamic model was developed and experimental data provided which demonstrated the feasibility of predicting the manometer's dynamic input from the manometer output by means of the generated model. This demonstration suggests a viable approach to eliminating substantially the manometer dynamics from the control-loop characteristics.

From experimental data, two representative models of the manometer were developed. For sma11 disturbances, a linear, second-order relation was found. For large dynamic input changes, a nonlinear first-order relationship was developed. The data supporting the models indicate the relatively large settling time and response lag to input pressure changes.

The manometer is a U-tube type with a built-in restriction in the base of the U. The gross effect of this restriction is to create drag on the flow of the mercury proportional to the square of the flow rate. In addition, analysis of experimental data indicates that this damping effect virtually dominates any inertial terms in the dynamics of the manometer.

Employing these empirically developed dynamic response equations, the feasibility of input pressure prediction was demonstrated by simulation of the response algorithm on experimental data with a computer (fig. 14) for a pressure change of about $1000 \mathrm{~N} / \mathrm{m}^{2}(20.9 \mathrm{psf})$. In al1 cases, the predicted input did remarkedly we11, especially in the presence of large rate of changes. The raggedness of the predicted signal is a result of insufficient quantization in the transient recorder. The eight-bit quantization allowed $\pm 10 \mathrm{~N} / \mathrm{m}^{2}(0.2 \mathrm{psf})$ uncertainty. This uncertainty plus the conversion uncertainty through the BCD digital-to-analog converter created a net $+14 \mathrm{~N} / \mathrm{m}^{2}(0.3 \mathrm{psf})$ uncertainty in recording $M$. Similar data for a sinusoida $\bar{I}, 0.16 \mathrm{~Hz}$, changing pressure is shown in figure 15 .

Experimental data and analysis have yielded a reasonable dynamic model for the manometer. From this model, experimental results have shown how the dynamic characteristic of the input can be estimated from the output of the manometer. By using better quantization, the prediction model should perform even better. With refined digital recording and analytical techniques, the nonlinear model coefficient could be more accurately identified.

The significance of employing a predictor is that the gross dynamic effect of the manometer is virtually removed from the closed-loop control. Directly, the control systems' bandwidth would be raised; thus, faster response to pressure parameter disturbances is allowed. In addition, the predictor could provide accurate pressure indication prior to the manometer having completely settled out. Either of the above-mentioned features would result in timeresponse improvement in the tunnel-data acquisition test point interarrival time. 
Other high accuracy instrumentation such as a11-electronic servoed fused quartz gages and resonating quartz crystal transducers will be evaluated to determine their applicability to this measurement problem.

\section{TEMPERATURE}

The basic technology covering temperature measurement over the NTF operating range is well established and offers a variety of applicable sensing techniques. Consequently, the principal effort will be directed at evaluating and selecting speciflc approaches tailored to specific measurement applications and developing an in-house expertise and application capability in the cryogenic temperature measurement domain.

The experience gained in temperature instrumentation of the Langley 0.3meter transonic cryogenic tunnel is a confident foundation for this effort. Nevertheless, problems such as sensor life and calibration accuracy might be anticipated.

\section{REFERENCES}

1. Yates, E. Carson, Jr.; and Gibson, Fredwick W.: Some Wind-Tunnel Measurements of Effects of Aeroelasticity on Transonic Aerodynamic Characteristics of a Supersonic Transport. NASA TM X-1098, 1965.

2. Bullock, Michael L.; and Warren, Richard E.: Electronic Total Station Speeds Survey Operations. Hewlett-Packard Journa1, April 1976, pp. 2-12.

3. Holmes, Harlan K.: Remote Displacement Sensor Using Microwave Modulated Light. Thesis, University of Virginia, August 1969. 


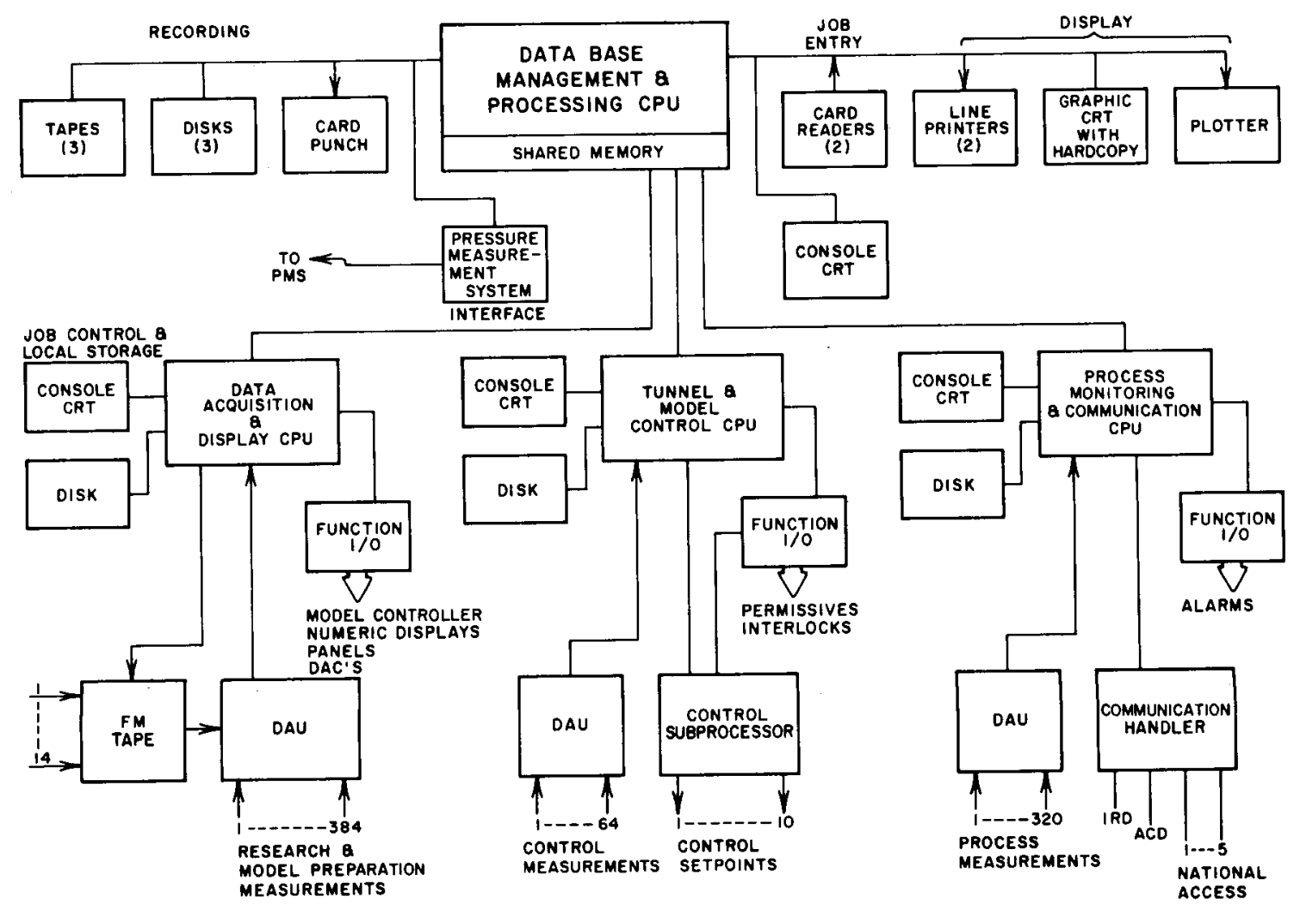

Figure 1.- NTF instrument complex.

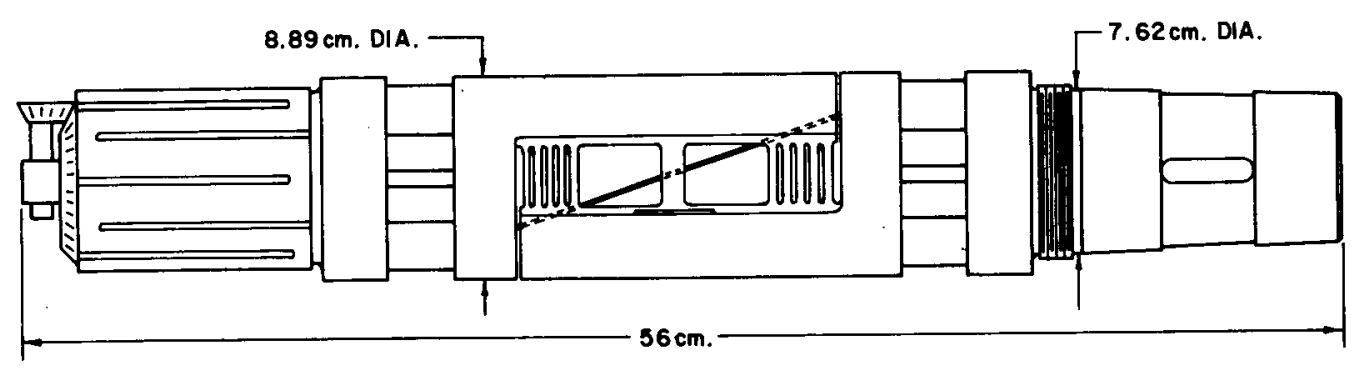

\begin{tabular}{lll}
\multicolumn{2}{l}{ SIMULTANEOUS LOADING } & \\
NORMAL, N & \pm 86736 & $(19.500 \mathrm{lb})$ \\
AXIAL, N & \pm 7784 & $(1750 \mathrm{lb})$ \\
PITCH, Nm & \pm 3389 & $(30000 \mathrm{ln}-\mathrm{lb})$ \\
ROLL, Nm & \pm 2260 & $(20000 \mathrm{in}-\mathrm{Ib})$ \\
YAW, Nm & \pm 1977 & $(17500 \mathrm{ln}-\mathrm{Ib})$ \\
SIDE, N & \pm 44480 & $(10000 \mathrm{lb})$
\end{tabular}

Figure 2.- NTF 86.7 kN (19 500 1b) balance. 


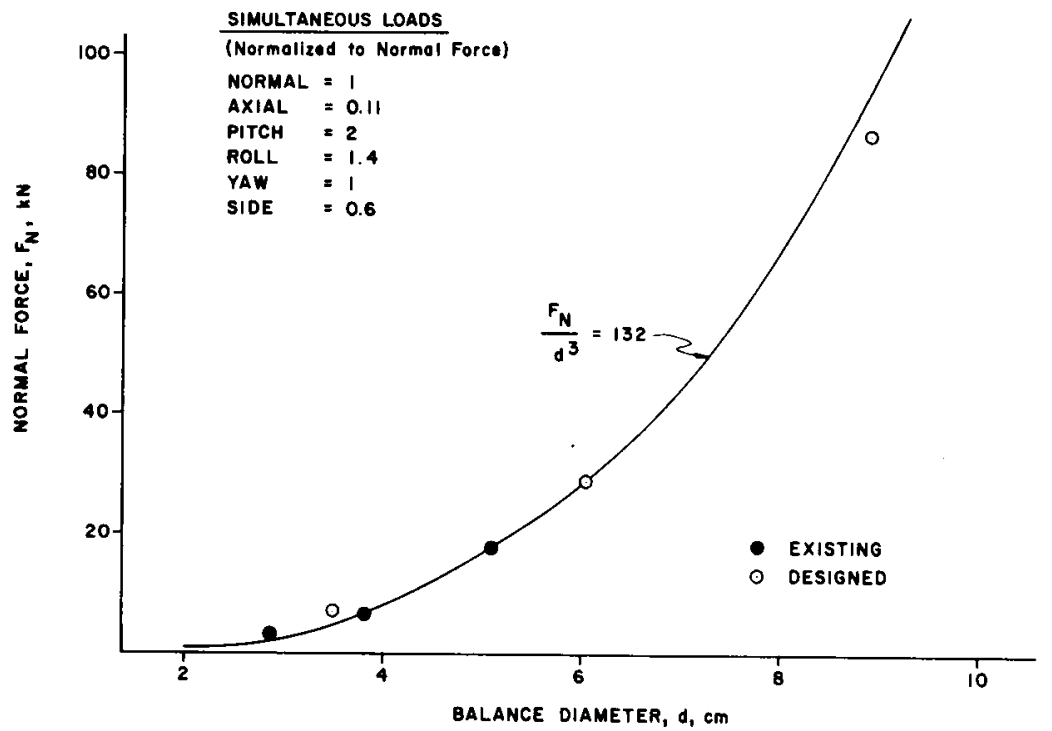

Figure 3.- Balance load as a function of diameter.

TEST CONDITIONS: $R=8.5 \times 10^{6}, M=0.8$

$T_{\infty}=114 \mathrm{~K}$, Dynamic pressure $=35.9 \mathrm{kN} / \mathrm{m}^{2}$ (750 psf)

BALANCE TEMPERATURES: O320K(117\% F) MAX. $\triangle T=16 \mathrm{~K}$

$0273 \mathrm{~K}\left(32^{\circ} \mathrm{F}\right) \quad \Delta T=19 \mathrm{~K}$

$\Delta 114 K\left(-254^{\circ} \mathrm{F}\right) " \quad \Delta T=4 K$
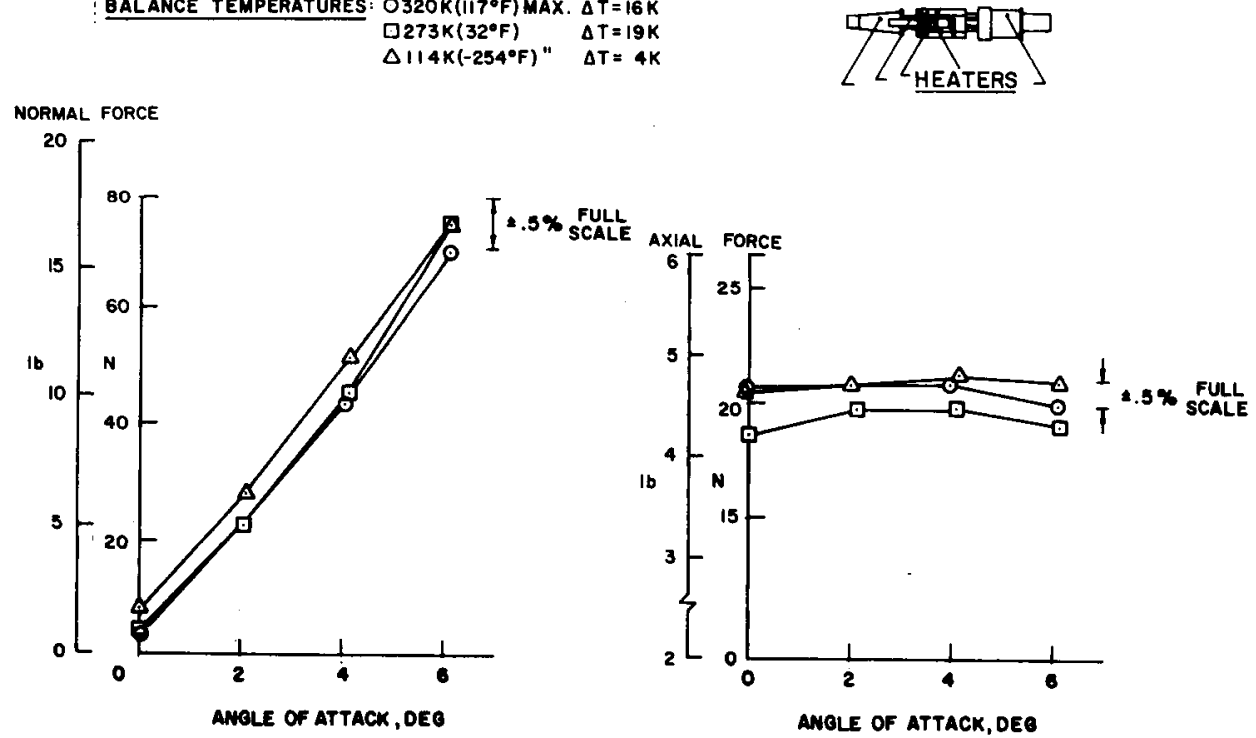

Figure 4.- 0.3-m transonic cryogenic tunnel data. 
NORMAL

SENSITIVITY,

$\mathrm{mV}$

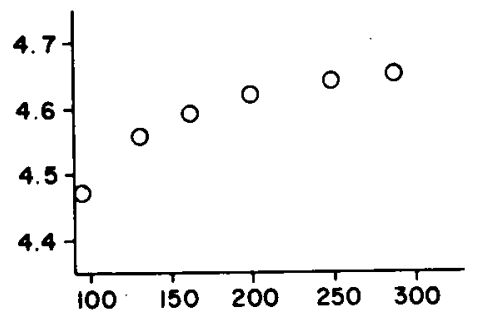

PITCH INTERACTION

ON NORMAL FORCE,

percent normal force

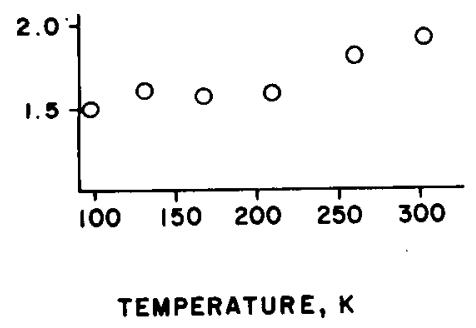

PITCH

SENSITIVITY,

$m V$

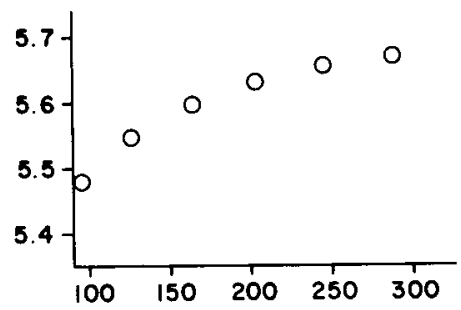

NORMAL FORCE INTERACTION ON PITCH, percent pltching moment

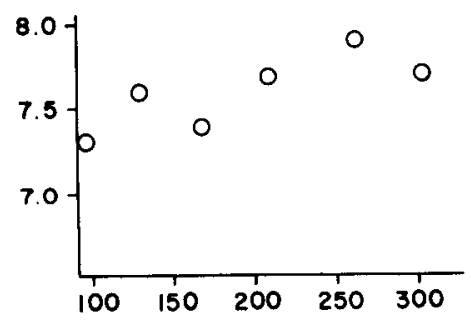

TEMPERATURE, $K$

Figure 5.- Balance laboratory calibration as a function of temperature.

\begin{tabular}{|l|c|c|c|c|}
\hline \multirow{2}{*}{ Material } & \multicolumn{2}{|c|}{ Yield strength $\left(\mathrm{MN} / \mathrm{m}^{2}\right)$ at -} & \multicolumn{2}{c|}{ Charpy impact strength, Nm at - } \\
\cline { 2 - 5 } & $298 \mathrm{~K}$ & $78 \mathrm{~K}$ & $298 \mathrm{~K}$ & $78 \mathrm{~K}$ \\
\hline Maraging 200 & 1427 & 1793 & 49 & 38 \\
Maraging 250 & 1758 & 2206 & 26 & 16 \\
Maraging 300 & 2000 & 2427 & 23 & 15 \\
Titanium alloy & 917 & 1475 & 52 & 19 \\
Beryllium & 1034 & 1275 & 8 & 8 \\
copper alloy & 1241 & 1675 & 41 & 5 \\
17-4PH & & &
\end{tabular}

Figure 6.- Materials under consideration for balance cryogenic application. 


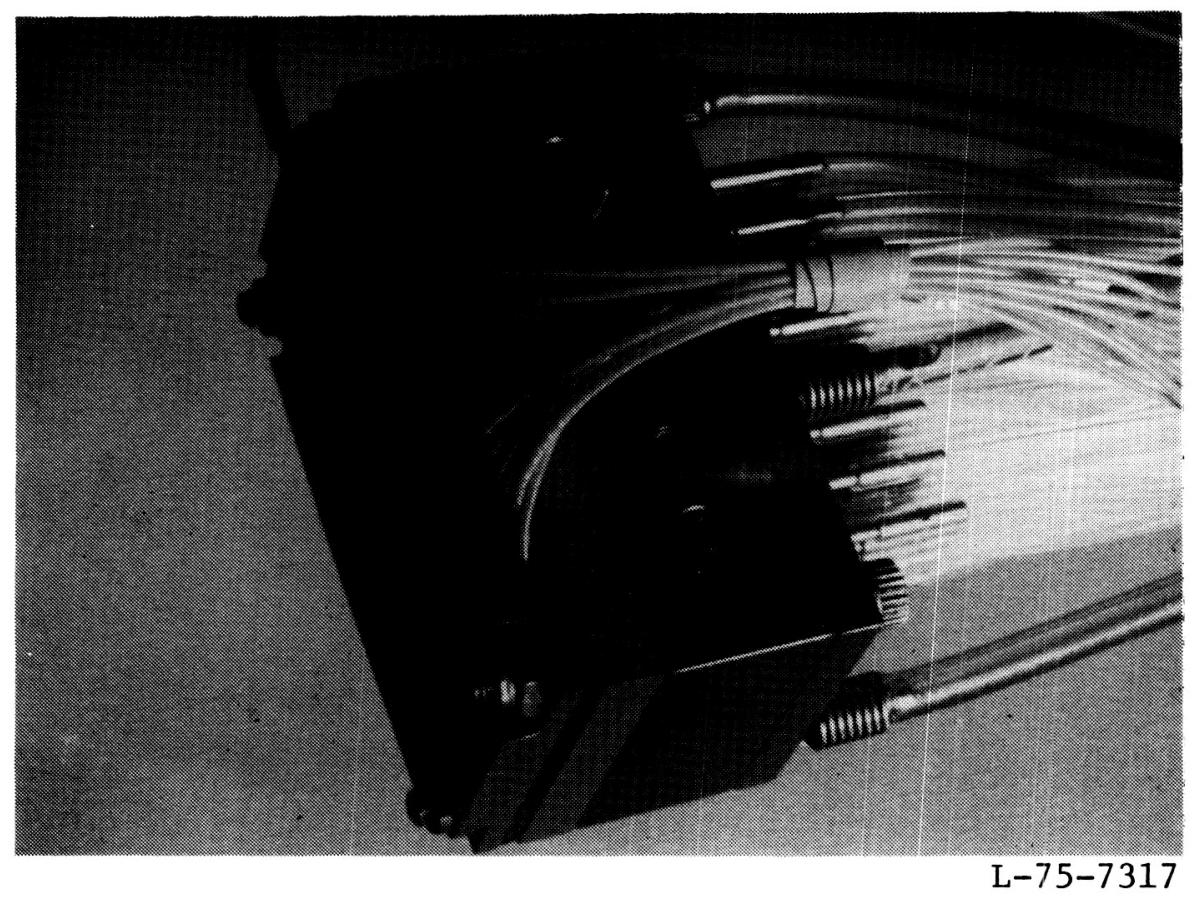

Figure 7.- 48-channel pressure sensor module.

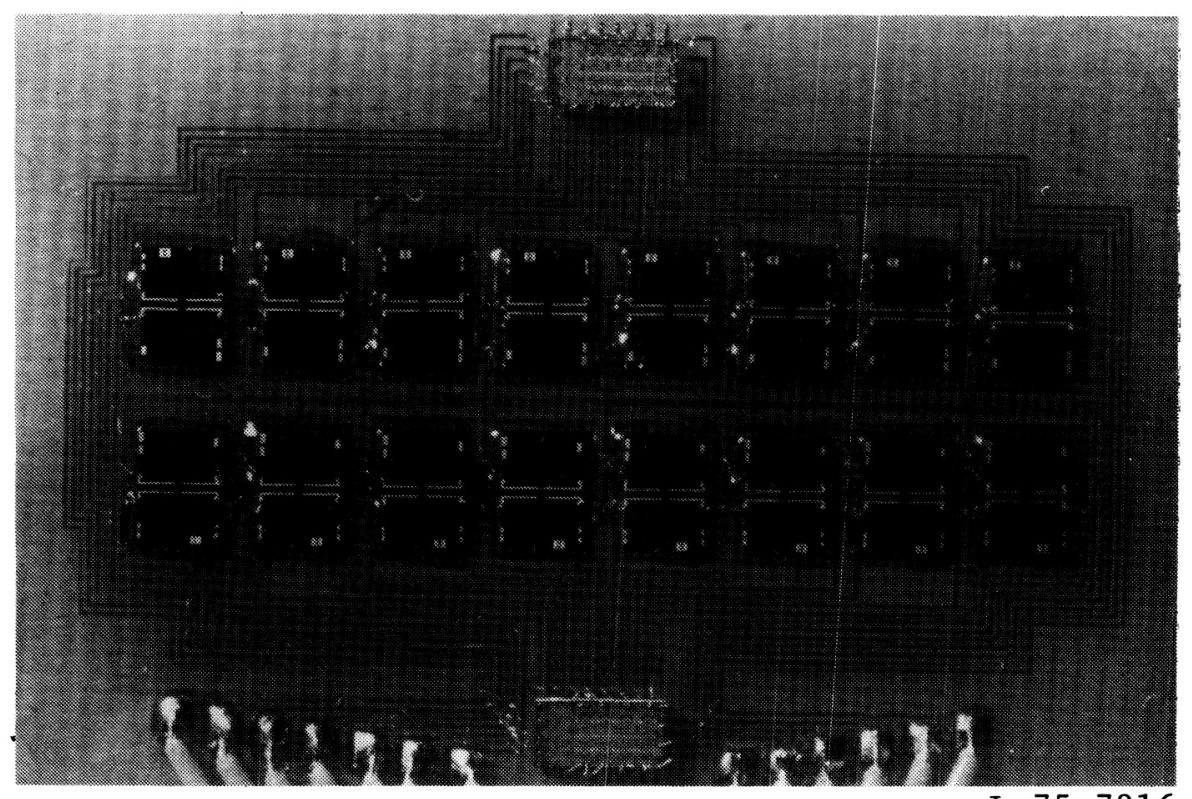

Figure 8.- Pressure sensor substrate board. 


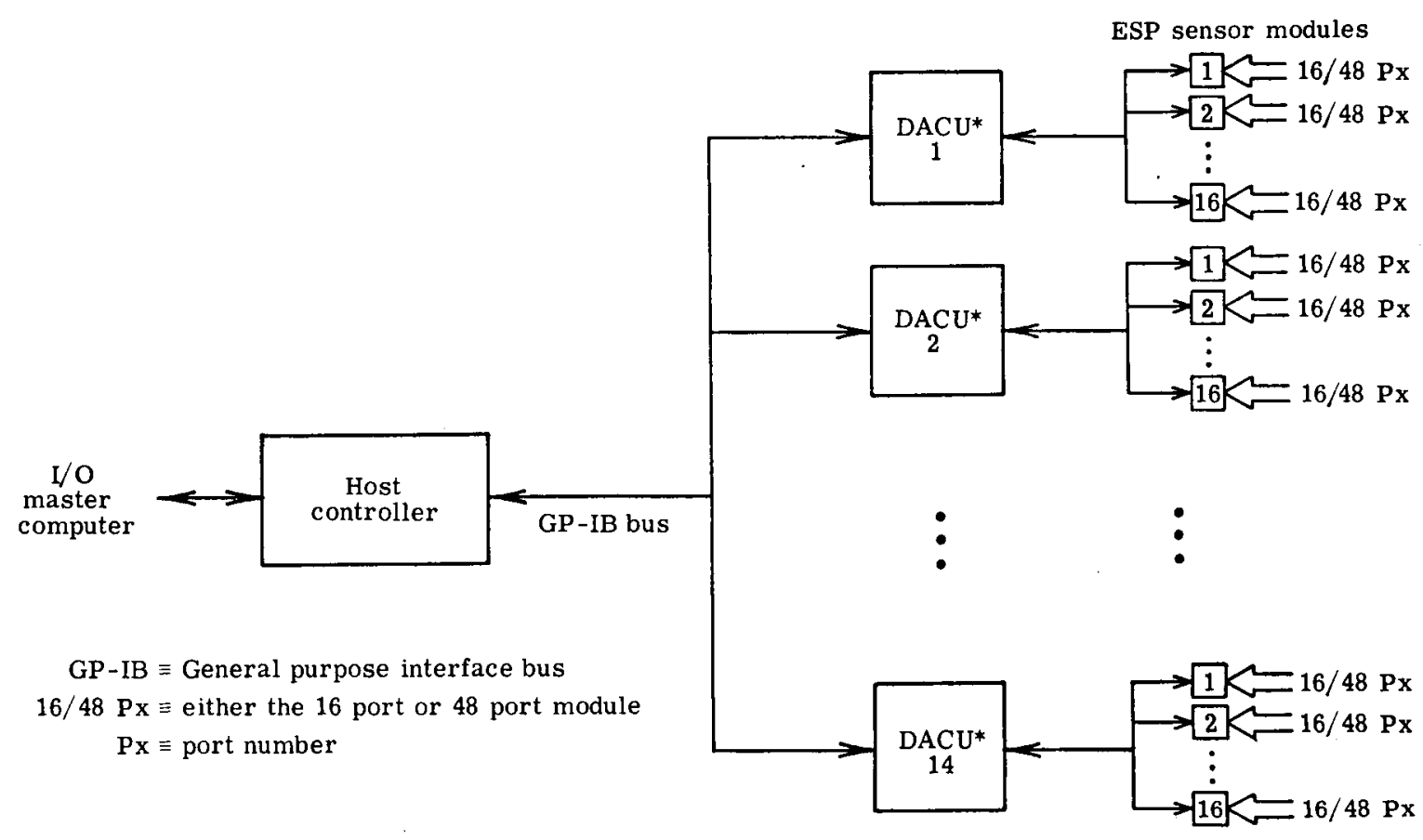

*Data acquisition and control unit

Figure 9.- Prototype electronic scanning pressure (ESP) measurement system.

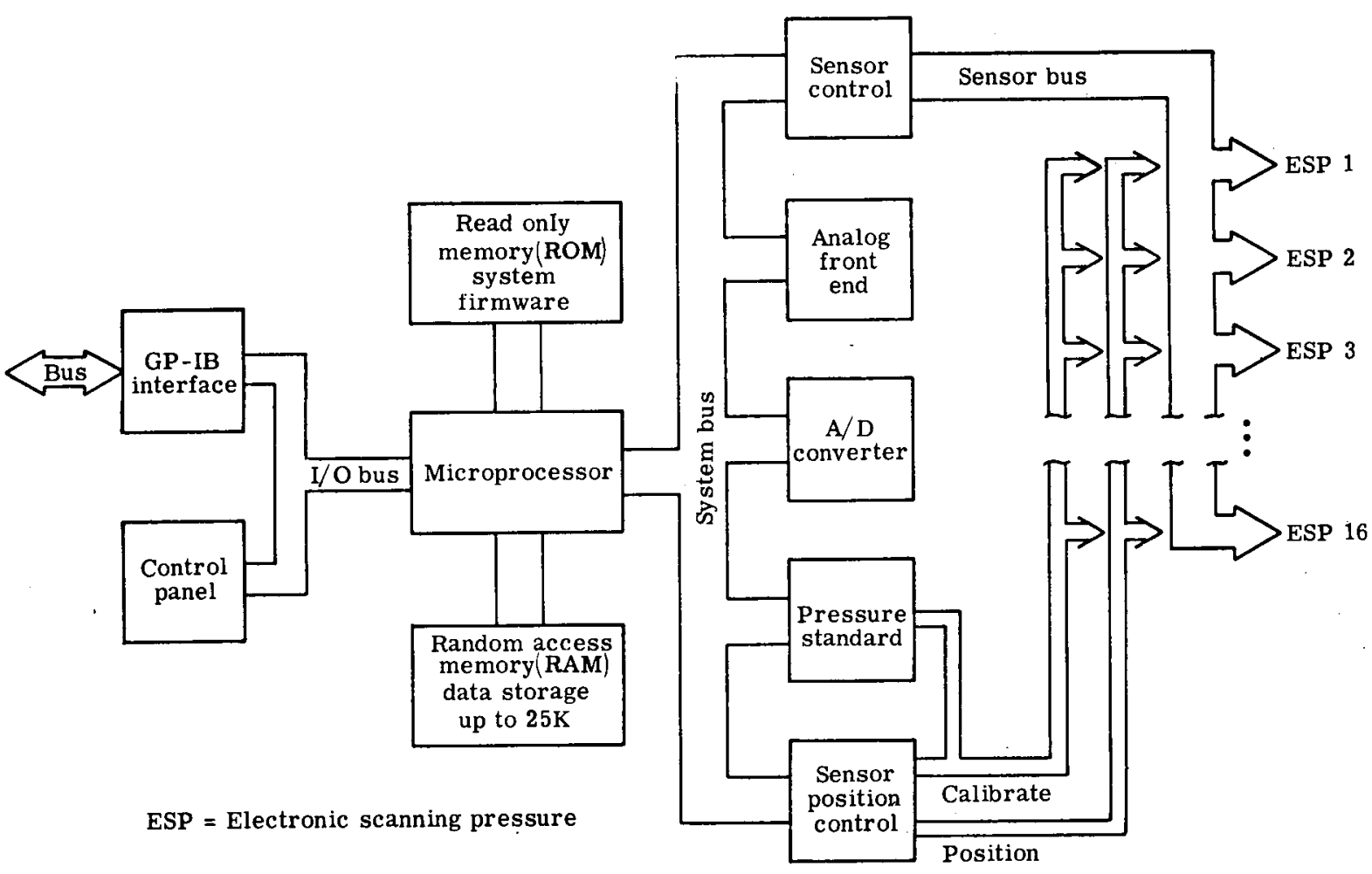

Figure 10.- Data acquisition and control unit (DACU). 
Total number of pressure ports

Number of DACU's

Number of channels/DACU

DACU data rate, measurement/sec

System data rate, measurement/sec

Maximum number data points/Pressure port

Lapse time between measurement on some port, msec

Calibration time, sec

Figure 11.- Typical operation of pressure measuring system for 300 and 1000 pressure ports.

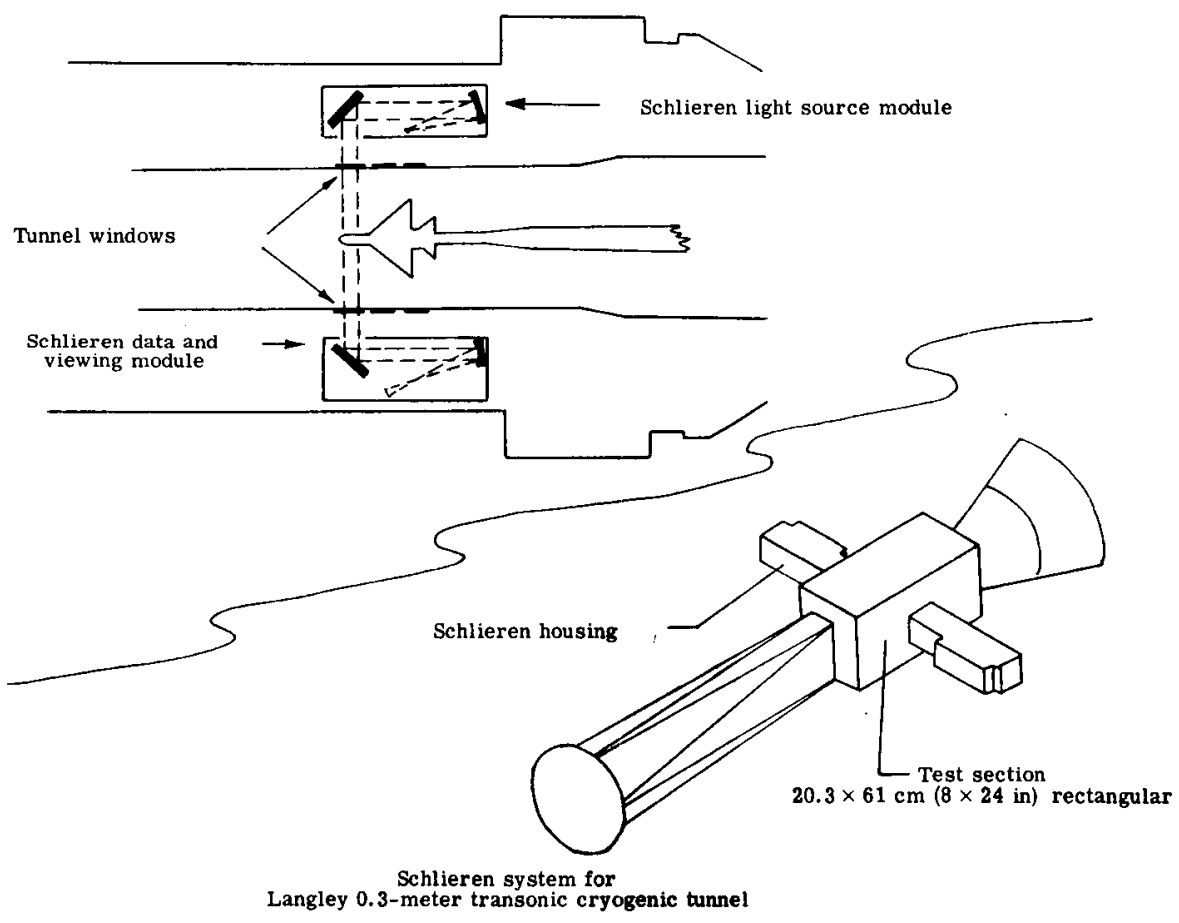

Figure 12.- NTF schlieren concept. 


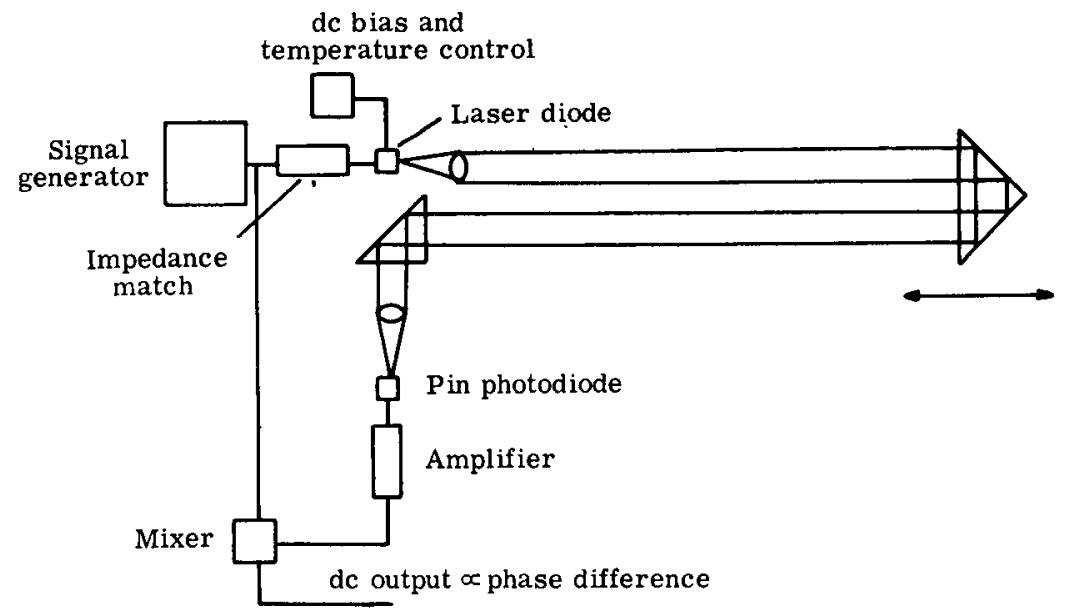

Figure 13.- Laboratory setup.

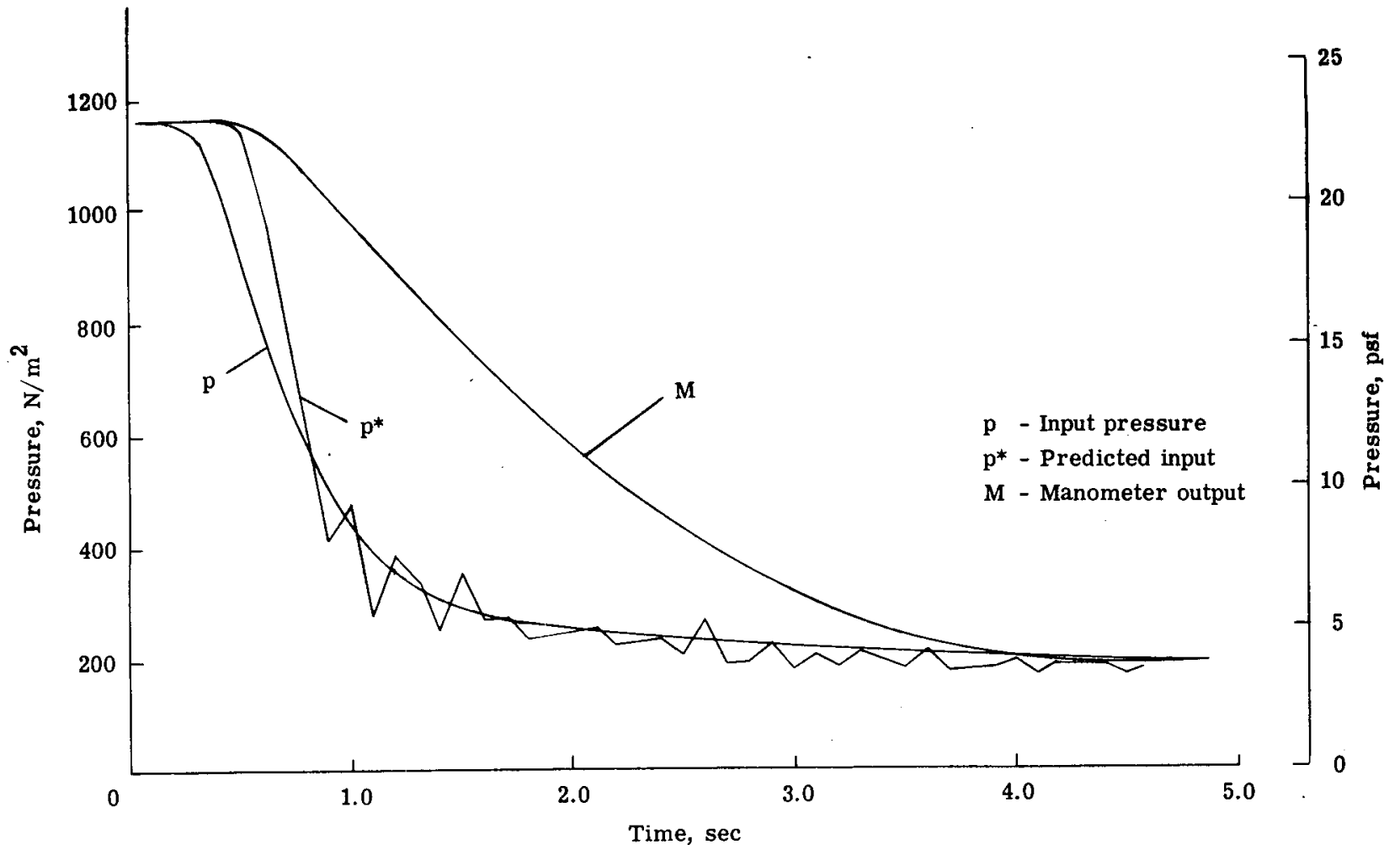

Figure 14.- Step-response data. 


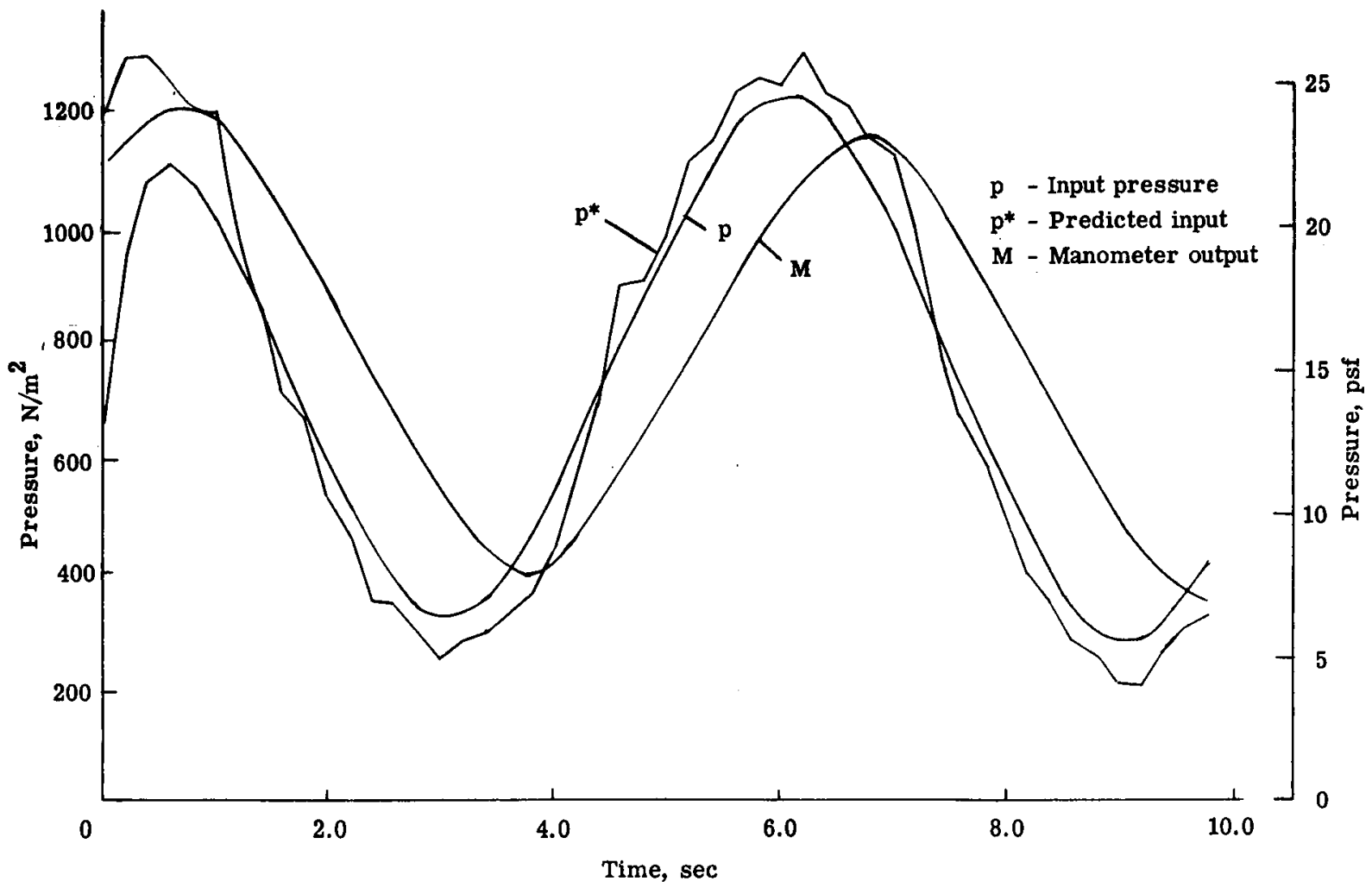

Figure 15.- Sinusoidal $(0.16 \mathrm{~Hz})$ response data. 